\title{
Gauge invariant 'monopoles' and color confinement mechanism
}

\section{Tsuneo Suzuki*}

Institute for Theoretical Physics, Kanazawa University, Kanazawa 920-1192, Japan and RIKEN, Radiation Laboratory, Wako 351-0158, Japan

E-mail: suzuki@hep.s.kanazawa-u.ac.jp

\section{Katsuya Ishiguro, Yoshifumi Nakamura and Toru Sekido}

Institute for Theoretical Physics, Kanazawa University, Kanazawa 920-1192, Japan and RIKEN, Radiation Laboratory, Wako 351-0158, Japan

\begin{abstract}
The dual Meissner effect is described and numerically observed in a gauge-invariant way in lattice Monte-Carlo simulations of pure $S U(2)$ QCD. A gauge-invariant monopole-like quantity on the lattice is defined by a gauge-invariant Abelian-like field strength. The Abelian-like field strength is expressed in terms of a unit-vector in color space which is constructed by a non-Abelian field strength itself. It is just equal to the absolute value of the corresponding non-Abelian field strength except for the sign. In this note we show the theoretical background and most numerical results will be published in a separate report [1] in this conference.
\end{abstract}

XXIIIrd International Symposium on Lattice Field Theory

25-30 July 2005

Trinity College, Dublin, Ireland

\footnotetext{
* Speaker.
} 


\section{Introduction}

One of the most essential problems of color confinement in QCD is to explain the mechanism of the flux squeezing of non-Abelian electric fields between a pair of static quark and antiquark. In $S U$ (2) QCD, $\left(E_{i}^{a}\right)^{2}$ or $\sqrt{\left(E_{i}^{a}\right)^{2}}$ is expected to be squeezed to reproduce the linear static potential. Numerically the expected squeezing of the gauge-invariant combination of the electric field was observed beautifully in lattice $S U(2)$ QCD [2].

Thirty years ago, 'tHooft [3] and Mandelstam [4] conjectured that the dual Meissner effect is the color confinement mechanism of QCD. However what causes the dual Meissner effect and how to treat the non-Abelian property were not clarified. An interesting idea is to utilize a topological monopole like the 'tHooft-Polyakov monopole [5, 6] found in $S U(2)$ QCD with an adjoint Higgs field $\phi$. A topological monopole has a bare magnetic charge satisfying the Dirac quantization condition with a bare electric charge. An important quantity is a 'tHooft Abelian-like field strength

$$
f_{\mu v}=n^{a} F_{\mu v}^{a}+\varepsilon_{a b c} n^{a}\left(D_{\mu} n\right)^{b}\left(D_{v} n\right)^{c}
$$

where $n^{a}$ is a unit vector in color space transforming as an adjoint representation and $\left(D_{\mu} n\right)^{b}$ is a covariant derivative. In $S U(2)$ QCD with a Higgs field, $\hat{\phi}^{a}$ is adopted as $n^{a}$, since there is a classical monopole solution corresponding to the choice.

However one may choose any adjoint operator for $n^{a}$ to discuss a topological monopole [7] in quantum field theory. This observation is important in real QCD without a Higgs field. We can discuss a topological monopole in terms of $n^{a}$ which is constructed in terms of gluon fields. A monopole picture can be seen more clearly if we project further $S U$ (3) QCD to an Abelian $U(1)^{2}$ theory by a partial gauge fixing [7]. After the Abelian projection, we have an Abelian $U(1)^{2}$ theory with Abelian electric and magnetic charges. It is conjectured in Ref. [7] that the condensation of the Abelian monopoles causes the dual Meissner effect explaining the color confinement. However there is a serious problem in this scenario. Namely there exist infinite ways of choosing $n^{a}$ or in other words infinite possible Abelian projections. Moreover, the monopole condensation, if happens, can explain only the squeezing of an Abelian-like electric field $f_{0 i}$ defined in Eq.(1.1). How good an approximation it is to the real and expected flux squeezing of $\sqrt{\left(E_{i}^{a}\right)^{2}}$ depends strongly on the choice of $n^{a}$.

An Abelian projection adopting a special gauge called Maximally Abelian gauge (MA) [8, 9, 10] is found to give us interesting results [11, 12, 13] supporting importance of the Abelian monopoles. In this case, the Abelian electric field can approximate very well the long-range behavior of the non-Abelian one, since other components are suppressed. However such beautiful results are not seen in other general gauges than the MA gauge.

It is the purpose of this note and the separate report [1] to show numerically that the dual Meissner effect occurs in a gauge-invariant way with the use of a gauge-invariant Abelian-like field strength and a monopole-like quantity. We do not need any Abelian projection nor any gaugefixing. In this note we explain the theoretical background of our idea and show most of numerical results in the separate report [1]. 


\section{Abelian-like field strength}

We define an Abelian-like field strength:

$$
f_{\mu v}(x)=\vec{n}_{\mu v}(x) \cdot \vec{F}_{\mu v}(x)
$$

where the summation over $\mu$ and $v$ is not taken ${ }^{1} . \vec{n}_{\mu \nu}$ is a unit vector in color space transforming as an adjoint representation in $\mathrm{SU}(2)$. Note that $f_{\mu \nu}$ is not a simple Lorentz tensor.

Explicitly we adopt the following unit vector in color space of $S U(2)$ QCD [14, 15, 16]:

$$
n_{\mu \nu}^{a}(x)=\varepsilon_{\mu \nu} \frac{F_{\mu \nu}^{a}(x)}{\sqrt{\sum_{a=1}^{3}\left(F_{\mu \nu}^{a}(x)\right)^{2}}},
$$

where $\varepsilon_{\mu \nu}$ is an antisymmetric tensor with the sign convention $\varepsilon_{\mu<v}=1$. The opposite sign convention can be adopted which means the existence of the sign ambiguity. The continuity could determine the relative sign. $F_{\mu \nu}^{a}$ is a non-Abelian field strength with a color charge $a$ and no summation is taken with respect to $\mu$ and $v$ in Eq.(2.2). This choice is unique in a sense that Eq.(2.1) is just equal to the gauge-invariant absolute value of the non-Abelian field strength itself except for the sign in $S U(2)$ QCD. Actually an electric field component $E_{i}$ defined by $f_{4 i}$ is $-\sqrt{\left(E_{i}^{a}\right)^{2}}$ the squeezing of which is to be explained.

A gauge-invariant monopole-like quantity is defined from the violation of the Bianchi identity:

$$
k_{\mu}(x)=\frac{1}{8 \pi} \varepsilon_{\mu v \alpha \beta} \partial_{v} f_{\alpha \beta}(x) .
$$

This is conserved but is not a simple Lorentz vector. Hereafter we call the monopole-like quantity simply as 'monopole'. We get from Eq.(2.3)

$$
\begin{aligned}
\vec{\nabla} \times \vec{E}+\partial_{4} \vec{B} & =4 \pi \vec{k}, \\
\vec{\nabla} \cdot \vec{B} & =-4 \pi k_{4},
\end{aligned}
$$

where

$$
\begin{aligned}
\vec{E} & \equiv\left(-\sqrt{\left(E_{1}^{a}\right)^{2}},-\sqrt{\left(E_{2}^{a}\right)^{2}},-\sqrt{\left(E_{3}^{a}\right)^{2}}\right), \\
\vec{B} & \equiv\left(\sqrt{\left(B_{1}^{a}\right)^{2}},-\sqrt{\left(B_{2}^{a}\right)^{2}}, \sqrt{\left(B_{3}^{a}\right)^{2}} .\right.
\end{aligned}
$$

Note that the magnetic charge defined in Eq.(2.5) does not satisfy the Dirac quantization condition with respect to bare charges contrary to the usual case of a magnetic charge defined in terms of a 'tHooft field strength.

\footnotetext{
${ }^{1}$ We used a little different Abelian-like field strength with an additional term as in 'tHooft field strength. However the additional term is not essential in the following discussions, since we are not dealing with a topological monopole. Hence we adopt the above definition (2.1) for simplicity.
} 


\section{3. 'Monopole' on the lattice}

Now we go to a lattice QCD framework. A non-Abelian field strength $F_{\mu v}(s)$ is given by a $1 \times 1$ plaquette variable defined by a path-ordered product of four non-Abelian link matrices on the lattice:

$$
U_{\mu v}(s)=\exp \left(i F_{\mu v}(s)\right)=U_{\mu v}^{0}(s)+i U_{\mu \nu}^{a}(s) \sigma^{a} .
$$

The unit vector in color space is

$$
n_{\mu v}^{a}(s)=\varepsilon^{\mu v} \frac{U_{\mu \nu}^{a}(s)}{\sqrt{1-\left(U_{\mu v}^{0}(s)\right)^{2}}},
$$

and the Abelian-like field strength is written similarly as in Eq.(2.1)

$$
f_{\mu \nu}(s)=n_{\mu \nu}^{a}(s) F_{\mu \nu}^{a}(s) .
$$

This definition is explicitly gauge-invariant.

We define a gauge-invariant lattice 'monopole' in the same way as in Eq.(2.3):

$$
k_{\mu}(s)=\frac{1}{8 \pi} \varepsilon_{\mu v \alpha \beta} \Delta_{v} f_{\alpha \beta}(s+\hat{\mu}),
$$

which satisfies $\Delta_{\mu}^{\prime} k_{\mu}(s)=0 . \Delta_{\mu}\left(\Delta_{\mu}^{\prime}\right)$ is a lattice forward (backward) derivative. Note that this 'monopole' is gauge-invariant and conserved but is not integer. In the separate report [1] we will see that the electric field $\sqrt{\left(E_{z}^{a}\right)^{2}}$ between a static quark pair is actually squeezed due to the solenoidal 'monopole current'. The dual Meissner effect can be seen in a gauge-invariant way.

\section{Comparison with Abelian monopoles after Abelian projections}

Here we compare our gauge-invariant 'monopole' with a (topological) monopole after an Abelian projection. In the latter case, an Abelian link variable $\theta_{\mu}^{A P}(s)$ is defined by a phase of the diagonal part of a non-Abelian link field after a gauge fixing. An Abelian field strength $\theta_{\mu \nu}^{A P}(s)$ is defined as $\theta_{\mu \nu}^{A P}(s) \equiv \theta_{\mu}^{A P}(s)+\theta_{v}^{A P}(s+\hat{\mu})-\theta_{\mu}^{A P}(s+\hat{v})-\theta_{v}^{A P}(s)$. An Abelian monopole is defined as [17]

$$
k_{\mu}^{A P}(s)=\frac{1}{8 \pi} \varepsilon_{\mu v \alpha \beta} \Delta_{v} \theta_{\alpha \beta}^{A P}(s+\hat{\mu}) .
$$

This is conserved and takes an integer number. Namely it is a topological monopole. It is known that, if we perform the MA gauge fixing where $\sum_{s, \mu} \operatorname{Tr}\left[U_{\mu}(s) \sigma_{3} U_{\mu}^{\dagger}(s) \sigma_{3}\right]$ is maximized, we get interesting results called as monopole dominance [11, 12, 13]. However, if we adopt a gaugefixing diagonalizing $F_{12}(s)$ ( $F_{12}$ gauge), such interesting results are not seen [11]. The $F_{12}$ gauge fixing on the lattice is defined in such a way as $U_{1}(s) U_{2}(s+\hat{1}) U_{1}^{\dagger}(s+\hat{2}) U_{2}^{\dagger}(s)$ is diagonalized.

We show numerical results of the correlations $C(r)$ between the 'monopole' and Abelian projected monopoles in Fig. 1

$$
C(r)=\frac{\left\langle\left|k_{\mu}^{A P}(0)\right|\left|k_{\mu}^{\prime}(r)\right|\right\rangle}{\left\langle\left|k_{\mu}^{A P}(0)\right|\right\rangle\left\langle\left|k_{\mu}^{\prime}(0)\right|\right\rangle}-1
$$




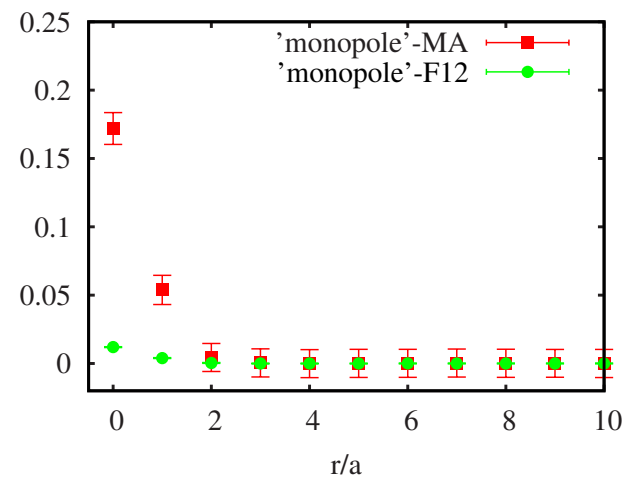

Figure 1: Correlation of the 'monopole' with Abelian projected monopoles in MA gauge and F12 gauge.

The above two kinds of Abelian monopoles are investigated. One of them is a monopole in the MA gauge fixing. It is interesting that the correlation is very strong. On the other hand the correlation is very weak between the gauge-invariant 'monopole' and an Abelian monopole in the $F_{12}$ gauge.

Note that the Abelian-like field strength Eq.(2.1) is reduced to an Abelian one if off-diagonal components are negligible. This occurs in the MA gauge and in the maximally Abelian Wilson loop gauge [18] where almost the same fine results as in the MA gauge are observed. In these cases, we can adopt $\vec{n}_{\mu \nu}=(0,0,1)$, since only the diagonal component exists. Hence the gaugeinvariant results we are going to show in the report [1] could explain why only restricted Abelian projection schemes like the MA gauge look nice among infinite possible candidates. In the case of the $F_{12}$ gauge, such a reduction does not occur.

\section{Conclusion}

We have defined a gauge-invariant monopole-like quantity by using an Abelian-like field strength on the lattice. This current is not a simple Lorentz vector and does not take an integer number. We have compared the gauge-invariant 'monopole' with Abelian topological monopoles appearing after Abelian projections. A strong correlation is observed between the gauge-invariant monopole and the Abelian monopole in the MA gauge, whereas no correlation is seen with the Abelian monopole in $F_{12}$ gauge. When the unit-vector $\vec{n}_{\mu \nu}$ in color space is well approximated as $\vec{n}_{\mu \nu}=(0,0,1)$, our gauge-invariant Abelian-like field strength becomes Abelian. Such a situation is expected in the MA gauge and the maximally Abelian Wilson loop gauge [18] where dominance of the topological Abelian monopole is seen.

In Ref. [1] we will show numerically that the dual Meissner effect occurs in a gauge-invariant way with the use of a gauge-invariant Abelian-like field strength and 'monopoles'. We do not need any Abelian projection nor any gauge-fixing.

\section{Acknowledgments}

The numerical simulations of this work were done using RSCC computer clusters in RIKEN. The authors would like to thank RIKEN for their support of computer facilities. T.S. is supported 
by JSPS Grant-in-Aid for Scientific Research on Priority Areas 13135210 and (B) 15340073.

\section{References}

[1] Toru Sekido, Katsuya Ishiguro, Yoshifumi Nakamura, and Tsuneo Suzuki. Gauge invariance of the abelian dual Meissner effect in pure SU(2) QCD, In Proceedings of Lattice 2005 conference, 2005.

[2] G. S. Bali, K. Schilling, and C. Schlichter. Observing long color flux tubes in SU(2) lattice gauge theory, Phys. Rev. D51:5165-5198, 1995, [hep-lat/9409005].

[3] G. 't Hooft. In A. Zichichi, editor, Proceedings of the EPS International, page 1225, 1976.

[4] S. Mandelstam. Vortices and quark confinement in non-Abelian gauge theories, Phys. Rept. 23:245-249, 1976.

[5] Gerard 't Hooft. Magnetic monopoles in unified gauge theories, Nucl. Phys. B79:276-284, 1974.

[6] Alexander M. Polyakov. Particle spectrum in quantum field theory, JETP Lett. 20:194-195, 1974.

[7] G. 't Hooft. Topology of the gauge condition and new confinement phases in non-Abelian gauge theories, Nucl. Phys. B190:455, 1981.

[8] Tsuneo Suzuki. Color confinement and asymptotic completeness, Prog. Theor. Phys. 69:1827, 1983.

[9] A. S. Kronfeld, M. L. Laursen, G. Schierholz, and U. J. Wiese. Monopole condensation and color confinement, Phys. Lett. B198:516, 1987.

[10] A. S. Kronfeld, G. Schierholz, and U. J. Wiese. Topology and dynamics of the confinement mechanism, Nucl. Phys. B293:461, 1987.

[11] Tsuneo Suzuki. Monopoles and confinement, Nucl. Phys. Proc. Suppl. 30:176-183, 1993.

[12] M. N. Chernodub and M. I. Polikarpov. Abelian projections and monopoles, In P. van Baal, editor, "Confinement, Duality and Nonperturbative Aspects of QCD", page 387, Cambridge.

[13] T. Suzuki. Low-energy effective theories from QCD, Prog. Theor. Phys. Suppl. 131:633-644, 1998.

[14] M. N. Chernodub, F. V. Gubarev, M. I. Polikarpov, and Valentin I. Zakharov. Dirac strings and monopoles in the continuum limit of SU(2) lattice gauge theory, Nucl. Phys. B592:107-128, 2001, [hep-th/0003138].

[15] M. N. Chernodub, F. V. Gubarev, M. I. Polikarpov, and Valentin I. Zakharov. Magnetic monopoles, alive, Phys. Atom. Nucl. 64:561-573, 2001, [hep-th/0007135].

[16] M. N. Chernodub, F. V. Gubarev, M. I. Polikarpov, and Valentin I. Zakharov. Towards abelian-like formulation of the dual gluodynamics, Nucl. Phys. B600:163-184, 2001, [hep-th/0 010265 ].

[17] T. A. DeGrand and D. Toussaint. Topological excitations and monte carlo simulation of Abelian gauge theory, Phys. Rev. D22:2478, 1980.

[18] F. Shoji, T. Suzuki, H. Kodama, and A. Nakamura. A new gauge fixing method for Abelian projection, Phys. Lett. B476:199-204, 2000, [hep-lat/9910042]. 